\title{
The Use of Novel Non-Vitamin K Antagonist Oral Anticoagulants Following Closure of the Left Atrial Appendage: Preliminary Results of Clinical Follow-Up
}

This article was published in the following Dove Press journal:

Drug Design, Development and Therapy

Jing Zhu

Jian Xu (D)

Department of Cardiology, The First Affiliated Hospital of University of Science and Technology of China, Hefei, 230000, People's Republic of China
Correspondence: Jian Xu

Department of Cardiology, The First Affiliated Hospital of University of Science and Technology of China, No. I of Tian E Hu Street, ShuShan District, Hefei, 230000, People's Republic of China

Tel +86 I36965456I0

Fax $+86 \quad 108316 \quad 1294$

Email xujianas88_dr@163.com
Objective: The aim of this study was to investigate the safety and efficacy of using novel non-vitamin $\mathrm{K}$ antagonist oral anticoagulants (NOACs) for anticoagulation following left atrial appendage closure (LAAC).

Methods: A total of 70 patients with non-valvular atrial fibrillation (NVAF) were enrolled in this study. All patients underwent successful LAAC between November 2018 and September 2019 in the Department of Cardiology of the First Affiliated Hospital of the University of Science and Technology of China. All patients subsequently completed a 45day postoperative follow-up period. Patients were grouped according to the postoperative anticoagulation regimen they received: there were 40 patients in the NOACs group and 30 patients in the warfarin group. Baseline clinical data, intraoperative data, and short-term postoperative follow-up data were collected and the two groups were compared.

Results: The intraoperative results showed no statistical difference between the two groups in respect of the occlusion rate, the compression ratio of the occluder, the volume of pericardial effusion, or the incidence of the following: residual shunts, device-related thromboses (DRT), stroke, and pericardial effusion ( $P>0.05$ in all cases). The residual shunt volume in the NOACs group was significantly smaller than that in the warfarin group $(P=0.04)$. During the 45 -day postoperative follow-up period, there was no statistical difference between the two groups in respect of the residual shunt volume, or the incidence of DRT, ischemic stroke and cerebral hemorrhage ( $P>0.05$ in all cases). Compared with the warfarin group, the residual shunt volume was significantly reduced in the NOACs group $(P=0.03)$. The incidence of minor hemorrhage and the total hemorrhage incidence in the NOACs group were significantly lower than those in the warfarin group ( $5 \%$ vs $30 \%, P=0.004$, and $5 \%$ vs $33.3 \%, P=0.002$, respectively).

Conclusion: In this study, the use of NOACs for postoperative anticoagulation therapy following LAAC did not increase the risk of embolization or hemorrhage during the shortterm follow-up period.

Keywords: non-valvular atrial fibrillation, left atrial appendage closure, novel non-vitamin $\mathrm{K}$ antagonist oral anticoagulants

\section{Introduction}

Non-valvular atrial fibrillation (NVAF) is a common clinical arrhythmia, and stroke and thromboembolic events are common and serious complications in patients with this condition. The incidence of stroke and thromboembolic events is two to seven times higher than in patients without atrial fibrillation (AF). In patients with AF, ischemic stroke accounts for $20-30 \%$ of all strokes, resulting in a serious economic burden on both families and society. ${ }^{1-3}$ Studies have shown that more than $90 \%$ of 
thrombi in NVAF patients originate from the left atrium, ${ }^{4}$ and that in preventing AF-related stroke, the therapeutic effects of left atrial appendage closure (LAAC) are noninferior to that of classical treatment with warfarin. ${ }^{5-7}$ Therefore, LAAC is an effective means of preventing stroke. For patients with AF who are at risk of stroke and cannot take long-term anticoagulant therapy, LAAC is an ideal supplement or alternative and has become a popular stroke prevention treatment for patients with AF worldwide. 8,9

Currently, there are no definitive guidelines or recommendations regarding anticoagulation therapy following LAAC. The commonly used clinical anticoagulation regimens all refer to the PROTECT-AF study. ${ }^{10,11}$ This study suggests the continuation of warfarin anticoagulation therapy for 45 days following surgery and discontinuation of clopidogrel after six months of dual antiplatelet therapy (DAPT), followed by replacement of DAPT with lifelong oral aspirin. Patients who are not suitable for warfarin anticoagulation can have DAPT with aspirin and clopidogrel. After six months of therapy, clopidogrel can be discontinued and replaced with lifelong oral aspirin as single drug therapy. However, there is no definitive clinical evidence for the use of the novel non-vitamin $\mathrm{K}$ antagonist oral anticoagulants (NOACs) following LAAC.

This study was a single-center, retrospective cohort study designed to preliminarily investigate the safety and efficacy of the oral NOACs following LAAC.

\section{Subjects and Methods Study Subjects}

A total of 74 patients who underwent LAAC in the arrhythmia subspecialty at the First Affiliated Hospital of the University of Science and Technology of China between November 2018 and September 2019 were enrolled in the study. In four patients, implantations failed due to a large orifice or the shallow depth of the left atrial appendage (LAA). The remaining 70 patients with NVAF underwent successful surgery with implantation of a Watchman occluder (Boston Scientific, US), and all patients completed a 45-day postoperative outpatient follow-up period. Patients were grouped according to the postoperative anticoagulation regimen they received: there were 40 patients in the NOACs group and 30 patients in the warfarin group. Sixteen for dabigatran and 24 for rivaroxaban (27-3 (not successfully implanted)). As the anticoagulant mechanism of the new oral anticoagulants differs from that of warfarin, the international normalized ratio (INR) is not used as the evaluation standard. For NOAC therapy either dabigatran or rivaroxaban were used: dabigatran $110 \mathrm{mg}$ bid, rivaroxaban $20 \mathrm{mg}$ bid (or $15 \mathrm{mg}$ bid if CRCI $<30-49 \mathrm{~mL} / \mathrm{min}$ ).

The study was conducted in accordance with the Declaration of Helsinki (revised 2013) and was approved by the Ethics Committee of the First Affiliated Hospital of the University of Science and Technology of China. All patients gave informed consent.

\section{Inclusion Criteria Were as Follows}

(1) age $\geq 18$ years, (2) a confirmed diagnosis of NVAF within the previous six months, (3) a HAS-BLED score of $\geq 3$, (4) a CHA2DS2-VAS score of $\geq 2$, (5) a contraindication to, unsuitability for, or poor compliance with NOACs, and (6) a contraindication to warfarin or the inability to take long term warfarin.

Following LAAC, all patients should receive oral anticoagulation with warfarin or NOACs as recommended and be followed up in outpatient clinic 45 days post-surgery.

\section{Exclusion Criteria Were as Follows}

(1) valvular AF, (2) AF caused by a reversible etiology, (3) thrombosis in the left atrium, (4) new onset ischemic stroke within the previous month, (5) other conditions necessitating the long-term use of anticoagulants, (6) life expectancy $<1$ year, (7) unobstructed foramen ovale or septal defect, and (8) left ventricular ejection fraction (LVEF) $<35 \%$ or patients in NYHA functional class IV and temporarily uncorrected. ${ }^{12-14}$

\section{Methods}

\section{Collection of Data}

A retrospective survey method was used to retrieve cases from the hospital information system that met the above criteria. The general characteristics of each patient were collected, including hospitalization ID, name, gender, age, surgery date, AF type, history of AF ablation, medical history (hypertension, diabetes, congestive heart failure, stroke, TIA, thromboembolism, hepatic and renal dysfunction, ischemic stroke, hemorrhage or hemorrhagic tendency), left atrium internal diameter, LVEF, CHA2DS2-VASc score, and HAS-BLED score. Microsoft Excel software was used to categorize and integrate the collected data and postoperative follow-up data with the composition ratios being analyzed. 
LAAC

All patients gave informed written consent for the surgery. Trans-esophageal echocardiography (TEE) was routinely performed to examine the morphology and structure of the LAA in order to exclude contraindications. ${ }^{15}$ The surgery was performed under general or local anesthesia and involved femoral vein puncture. The long sheath was implanted, and the guidewire was delivered through a septal puncture under TEE guidance. The long sheath was then replaced with a $14 \mathrm{~F}$ guiding sheath. The diameter of the LAA, the depth of the LAA, and the size of the anchoring area were measured by TEE or intra-cardiac echocardiography. The appropriate Watchman LAA occluder was selected, implanted under real-time ultrasonography guidance, and released once it was in place. The pull test was conducted to verify firm anchoring of the occluder. Finally, the existence of residual blood flow around the occluder and the presence of a pericardial effusion was identified. If the condition was in accordance with the PASS principle, the occluder was released, and the patient was transferred to CCU.

\section{The Intraoperative and Postoperative Anticoagulation Therapy: All Patients Received a Continuous Anticoagulation Regimen}

During the operation, heparin was used for anticoagulation: a loading dose of $100 \mathrm{U} / \mathrm{kg}$ was given followed by an infusion of $1000 \mathrm{U} / \mathrm{h}$. The activated coagulation time was monitored dynamically and maintained within the range of 250 300 s. Six hours after the operation, low molecular weight heparin was given based on body weight. For those without contraindications for warfarin, low molecular weight heparin was discontinued when the INR reached 1.5 and warfarin was continued with the INR maintained at 2.0 3.0. For those who could not take warfarin orally, dabigatran $110 \mathrm{mg}$ bid or rivaroxaban $15 \mathrm{mg}$ once daily was given as a single replacement drug. All patients were followed up 45 days after the operation. In cases where no residual shunt or thrombosis on the surface of the occluder was identified, the warfarin or NOACs were discontinued and the patient was started on DAPT (aspirin $100 \mathrm{mg}$ QD + clopidogrel $75 \mathrm{mg}$ QD) up until six months post-surgery. Aspirin was then given as oral monotherapy.

\section{The 45-Day Postoperative Follow-Up}

Routine follow-up visits were conducted in the outpatient clinic 45 days after LAAC. The clinical medical history was reviewed, including whether there had been any adverse events such as ischemic stroke, various hemorrhages, or device-related thrombosis (DRT). Color Doppler echocardiography and an electrocardiogram were performed and coagulation function was tested.

\section{Observation Indicators}

During the operation, the occlusion rate, the compression ratio of the occluder, the fall-off of the device, the residual shunt volume (if present), the volume of pericardial effusion (if present), the formation of DRT, along with other complications including stroke and vascular injury were observed. At 45 days following surgery, the formation of DRT, residual shunts and the volume of the shunts, stroke and its complications (including digestive, intracranial, nasal, or gum hemorrhage), subcutaneous hematoma, and mucosal ecchymosis were noted.

\section{Statistical Analysis}

SPSS 18.0 software was used for data analysis. The measurement variables conforming to the normal distribution were expressed by $(\mathrm{x} \pm \mathrm{s})$, and independent sample $t$-tests were used for comparison between groups. The continuous variables that did not conform to the normal distribution were represented by the median (the minimum, the maximum), and the rank-sum test was used for comparison between groups. $P<0.05$ was considered statistically significant.

\section{Results}

\section{Comparison of the Baseline Characteristics Between the Two Groups (Table I)}

There was no statistical difference in age, gender, history of hypertension, stroke, TIA, thromboembolism, vascular disease, congestive heart failure, diabetes mellitus, hepatic and renal dysfunction, stroke and hemorrhage, unstable INR, CHA2DS2-VASc scores, HAS-BLED scores, the inner diameter of the left atrium, LVEF, the diameter of the LAA orifice, and the depth of the LAA between the two groups $(P>0.05$ in all).

\section{Comparison of the Intraoperative Data Between the Two Groups (Table 2)}

There was no statistically significant difference in the occlusion rate, the compression ratio of the occluder, the incidence of residual shunts and DRT, the incidence of 
Table I Comparison of Baseline Data Between the Two Groups

\begin{tabular}{|c|c|c|c|}
\hline Items & $\begin{array}{c}\text { The NOACs Group } \\
\text { (40 Cases) }\end{array}$ & $\begin{array}{c}\text { The Warfarin Group } \\
\text { (30 Cases) }\end{array}$ & $p$ value \\
\hline Age [Year, $M$ (The maximum, the minimum)] & $67(80,50)$ & $65(86,46)$ & 0.38 \\
\hline Male [Case (\%)] & $2 I(52.5)$ & $20(66.7)$ & 0.20 \\
\hline Hypertension [Case (\%)] & $24(60)$ & $24(80)$ & 0.85 \\
\hline Diabetes mellitus [Case (\%)] & $10(25)$ & $4(13.3)$ & 0.23 \\
\hline History of stroke [Case (\%)] & $3 \mid(77.5)$ & $21(70)$ & 0.48 \\
\hline Congestive heart failure [Case (\%)] & $3(7.5)$ & $\mathrm{I}(3.3)$ & 0.46 \\
\hline History of vascular diseases & $5(12.5)$ & $6(20)$ & 0.40 \\
\hline Stroke/TIA/Thromboembolism[Case (\%)] & $3 I(77.5)$ & $22(73.3)$ & 0.69 \\
\hline History of hemorrhage [Case (\%)] & $5(12.5)$ & $4(13.3)$ & 0.92 \\
\hline History of unstable INR & $10(25)$ & $4(13.3)$ & 0.23 \\
\hline Combination of aspirin or non-steroidal anti-inflammatory drugs & $2 I(52.5)$ & $17(56.7)$ & 0.73 \\
\hline Alcoholism & $\mathrm{I}(2.5)$ & $\mathrm{I}(3.3)$ & 0.84 \\
\hline CHA2DS2-VASc scores & $4.05 \pm 1.09$ & $3 \pm 1.37$ & 0.86 \\
\hline HAS-BLED scores & $3.18 \pm 0.59$ & $3.17 \pm 0.82$ & 0.96 \\
\hline The inner diameter of the left atrium & $47.23 \pm 5.66$ & $49.07 \pm 7.43$ & 0.25 \\
\hline LVEF & $64.45 \pm 9.43$ & $62.4 \pm 8.24$ & 0.35 \\
\hline The diameter of the left atrial appendage orifice & $25.28 \pm 4.27$ & $23.93 \pm 3.68$ & 0.18 \\
\hline Length of the left atrial appendage & $26.63 \pm 2.60$ & $25.13 \pm 3.75$ & 0.06 \\
\hline Atrial fibrillation [Case (\%)] & $30(75)$ & $19(63.3)$ & 0.42 \\
\hline Chronic renal dysfunction (creatinine clearance rate $<80 \mathrm{~mL} / \mathrm{min}$ ) [Case (\%)] & $3(7.5)$ & $5(16.7)$ & 0.40 \\
\hline Coronary heart disease & $8(20)$ & $7(23.3)$ & 0.36 \\
\hline
\end{tabular}

stroke and pericardial effusion, and the volume of effusion between the two groups ( $P>0.05$ in all). The volume of residual shunts in the NOACs group was significantly lower than that in the warfarin group $(P=0.04)$.

\section{Comparison of the Data During the 45-Day Postoperative Follow-Up} Between the Two Groups (Table 3)

There was no significant difference in the incidence of DRT, the volume of residual shunts, ischemic stroke incidence, and hemorrhagic stroke between the two groups
$(P>0.05$ in all). The volume of the residual shunt in the NOACs group was significantly lower than that in the warfarin group $(P=0.034)$. The incidence of minor hemorrhage and the incidence of total hemorrhage in the NOACs group were significantly lower than those in the warfarin group (5\% vs $30 \%, P=0.004$, and $5 \%$ vs $33.3 \%$, $P=0.002$, respectively).

\section{Discussion}

Currently, the prevalence of AF in China is approximately $0.77 \% .^{15,16}$ The prevalence and incidence of AF increases

Table 2 Intraoperative Data of the Two Groups Were Compared

\begin{tabular}{|c|c|c|c|}
\hline Items & $\begin{array}{c}\text { The NOACs Group } \\
\text { (40 Cases) }\end{array}$ & $\begin{array}{c}\text { The Warfarin Group } \\
\text { (30 Cases) }\end{array}$ & $p$ value \\
\hline The compression ratio of the occluder $(\%, x \pm s)$ & $16.20 \pm 4.78$ & $15.56 \pm 4.38$ & 0.71 \\
\hline The occlusion rate [Case (\%)] & $40(100)$ & $29(96.7)$ & $>0.99$ \\
\hline The residual shunts [Case (\%)] & $5(12.5)$ & $8(26.7)$ & 0.12 \\
\hline The volume of the residual shunts $[\mathrm{mm}, \mathrm{M}$ (the maximum, the minimum)] & $0(0,3)$ & $0(0,4.5)$ & 0.04 \\
\hline Device-related thrombosis [Case (\%)] & $0(0)$ & $\mathrm{I}(3.3)$ & 0.25 \\
\hline Stroke [Case (\%)] & $\mathrm{I}(2.5)$ & $0(0)$ & 0.39 \\
\hline Pericardial effusion [Case (\%)] & $\mathrm{I}(2.5)$ & $2(6.6)$ & 0.40 \\
\hline The volume of the pericardial effusion [mm, $M$ (the maximum, the minimum)] & $0(0,5)$ & $0(0,11)$ & 0.31 \\
\hline
\end{tabular}

Abbreviation: NOACs, novel oral anticoagulants. 
Table 3 Comparison of Follow-Up Data 45 Days After Operation Between the Two Groups

\begin{tabular}{|c|c|c|c|}
\hline Items & $\begin{array}{c}\text { The NOACs Group } \\
\text { (40 Cases) }\end{array}$ & $\begin{array}{c}\text { The Warfarin Group } \\
\text { (30 Cases) }\end{array}$ & $p$ value \\
\hline The residual shunts [Case (\%)] & $3(7.5)$ & $7(23.3)$ & 0.06 \\
\hline The volume of the residual shunts [mm, $M$ (the maximum, the minimum)] & $0(0,2)$ & $0(0,3)$ & 0.03 \\
\hline Device-related thrombosis [Case (\%)] & $I(2.5)$ & $2(6.7)$ & 0.4 \\
\hline Ischemic stroke [Case (\%)] & $\mathrm{I}(2.5)$ & $0(0)$ & 0.39 \\
\hline Cerebral hemorrhage [Case (\%)] & $0(0)$ & $I(3.3)$ & 0.25 \\
\hline Minor hemorrhage [Case (\%)] & 2(5) & $9(30)$ & 0.004 \\
\hline Total hemorrhage [Case (\%)] & 2(5) & $10(33.3)$ & 0.002 \\
\hline
\end{tabular}

progressively with age. Strokes and thromboembolic events are both common and serious complications of AF. Anticoagulation therapy can significantly reduce the incidence of both stroke and systemic embolic events, thereby improving prognosis. LAAC is non-inferior to classical warfarin therapy in preventing AF-related stroke. The 2018 Chinese guidelines for the diagnosis and treatment of $\mathrm{AF}^{3}$ also recommend LAAC as an intervention for patients with NVAF who have contraindications to longterm anticoagulation therapy and are at high risk of stroke. Anticoagulation therapy is necessary following LAAC to prevent DRT. A multicenter review by Fauchier et $\mathrm{al}^{17}$ revealed a $7.2 \%$ incidence of DRT following occlusion. The PROTECT-AF study suggested that the incidence of DRT was $4.2 \%(2.4 \% \sim 6.8 \%){ }^{18,19}$ In this study, one case of DRT was identified in the warfarin group from the intraoperative data. During the 45-day postoperative follow-up period, the incidence of DRT was $4.3 \%$, with one case in the NOACs group and two cases in the warfarin group. There was no statistical difference between the two groups. There was a correlation between the formation of DRT following occlusion and patient factors, inadequate postoperative anticoagulation therapy, and the device adopted.

Currently, there are differences in global anticoagulation regimens following $\mathrm{LAAC}$, although it is agreed that patients should continue to take warfarin for anticoagulation for 45 days after the procedure. ${ }^{20}$ However, there are many problems associated with the oral administration of warfarin including its narrow therapeutic window and the fact that food and medication can affect its efficacy and safety. Consequently, monitoring the coagulation effect of warfarin using the INR and implementing an individualized drug regimen is necessary. ${ }^{21}$ Moreover, the risk of hemorrhage is high with warfarin therapy, and this increases with age. In recent years, a series of NOACs have emerged providing a new option for clinical anticoagulation therapy. ${ }^{22}$ Clinical studies have verified that NOACs are equally effective and safer than warfarin in preventing thromboembolism in patients with $\mathrm{AF}^{23,24}$

However, there is no clear evidence on the safety and efficacy of NOACs in preventing DRT following LAAC. Related studies have shown that the incidence of thromboembolic events is reduced. There is no difference in hemorrhagic events when NOACs are used following LAAC as compared with the traditional postoperative anticoagulation regimen of warfarin. ${ }^{10} \mathrm{~A}$ multicenter, observational study conducted by Fountain et al showed no thromboembolic events, such as cerebral infarction or atrial thrombosis, in the group implanted with the Watchman occluder as compared with the group implanted with the ACP occluder. Compared to the warfarin group, the dabigatran group had a lower incidence of hemorrhagic events $(18.2 \%$ vs $2.9 \%$, respectively, $P=0.04)$, while the rivaroxaban group showed a tendency towards a lower incidence of hemorrhagic events $(0 \%, P=0.08)$. A subgroup analysis of the EWOLUTION study suggested no correlation between the occurrence of postoperative hemorrhagic events after LAAC and the application of anticoagulation protocols. However, the lowest incidence of postoperative hemorrhagic events occurred in the oral NOACs group. A small number of foreign studies have compared different anticoagulation protocols following LAAC and found no statistically significant differences in the incidences of DRT formation or embolic events between the different anticoagulation protocols. When investigating patients who underwent a 45-day postoperative follow-up with LAAC, Bösche et $\mathrm{al}^{25}$ found that no stroke events occurred in patients treated with early NOACs therapy and those treated with aspirin and clopidogrel. A retrospective study by Enomoto et $\mathrm{al}^{26}$ found no statistically significant differences in the incidences of 
device surface thrombosis, thromboembolic events, or hemorrhagic events between patients anti-coagulated with NOACs and those treated with oral warfarin anticoagulation following LAAC. The efficacy and safety of NOACs for percutaneous LAAC remains poorly reported in China. In this study, when the intraoperative data were compared between the two groups, one case of stroke was identified in the NOACs group and none in the warfarin group. At 45-day follow-up, one case of ischemic stroke was identified in the NOACs group and none in the warfarin group. Results also showed that the residual shunt volume was significantly reduced in the NOACs group as compared with the warfarin group, which might contribute to the reduced risk of embolization and hemorrhage. Compared with the data in the warfarin group, the incidences of minor small hemorrhagic events and total hemorrhagic events were significantly reduced in the NOACs group, and the incidence of adverse events was significantly lower. The results during the follow-up period were generally consistent with the above studies.

In this study, limitations included the small sample size and the short duration of follow-up. Larger sample sizes and long-term follow-up data are needed in the future to support the validity and safety of NOACs following LAAC.

\section{Conclusion}

In this study, the use of NOACs for postoperative anticoagulation therapy following LAAC did not increase the risk of embolization or hemorrhage during the short-term follow-up period.

\section{Acknowledgments}

We are particularly grateful to all the people who have given us help on our article.

\section{Funding}

There is no funding to report.

\section{Disclosure}

The authors declare that they have no competing interests.

\section{References}

1. Kirchhof P, Benussi S, Kotecha D, et al. 2016 ESC guidelines for the management of atrial fibrillation developed in collaboration with EACTS [published correction appears in rev esp cardiol (Engl Ed). 2017 Nov;70(11):1031]. Rev Esp Cardiol. 2017;70(1):50. doi:10.1016/ j.rec.2016.11.033
2. Guérios ÊE, Chamié F, Montenegro M, et al. First results of the Brazilian registry of percutaneous left atrial appendage closure. Arq Bras Cardiol. 2017;109(5):440-447. doi:10.5935/abc.20170150

3. Huang CX, Zhang Z, Huang DJ, et al. Current knowledge and management recommendations of atrial fibrillation: 2018. Chin J Cardiac Arrhythmias. 2018;22(4):279-346.

4. Yang B, Yang Q, Xie Q. Predictive factors of recurrence of atrial fibrillation in patients accepting catheter ablation. J Pract Med. 2016;32(22):3717-3720.

5. Saw J, Fahmy P, Azzalini L, et al. Early Canadian multicenter experience with WATCHMAN for percutaneous left atrial appendage closure. J Cardiovasc Electrophysiol. 2017;28(4):396-401. doi: $10.1111 /$ jce. 13168

6. Acampa M, Lazzerini PE, Guideri F, Tassi R, Lo Monaco A, Martini G. Inflammation and atrial electrical remodelling in patients with embolic strokes of undetermined source. Heart Lung Circ. 2019;28(6):917-922. doi:10.1016/j.hlc.2018.04.294

7. Boriani G, Pieragnoli P, Botto GL, et al. Effect of PR interval and pacing mode on persistent atrial fibrillation incidence in dual chamber pacemaker patients: a sub-study of the international randomized MINERVA trial. Europace. 2019;21(4):636-644. doi:10.1093/europace/euy286

8. Reddy VY, Doshi SK, Kar S, et al. 5-year outcomes after left atrial appendage closure: from the PREVAIL and PROTECT AF trials. $J$ Am Coll Cardiol. 2017;70(24):2964-2975. doi:10.1016/j. jacc.2017.10.021

9. Zhong MZ. Current state and outlook in the diagnosis and treatment of atrial fibrillation. J Pract Med. 2015;31(6):872-875.

10. Fountain RB, Holmes DR, Chandrasekaran K, et al. The PROTECT AF (WATCHMAN left atrial appendage system for embolic PROTECTion in patients with atrial fibrillation) trial. Am Heart $J$. 2006;151(5):956-961. doi:10.1016/j.ahj.2006.02.005

11. Bergmann MW, Betts TR, Sievert H, et al. Safety and efficacy of early anticoagulation drug regimens after WATCHMAN left atrial appendage closure: three-month data from the EWOLUTION prospective, multicentre, monitored international WATCHMAN LAA closure registry. EuroIntervention. 2017;13(7):877-884. doi:10.4244/EIJ-D-17-00042

12. Jiang C, Zhang Y, Li SL, et al. Efficacy and safety of percutaneous left atrial appendage occlusion for secondary stroke prevention in patients with atrial fibrillation. Chin Med. 2018;33(01):01-04.

13. Chen YY, Chen YH, Zhang YH, et al. Use of novel oral anticoagulant left atrial appendage closure: results of peri-operative and short-term clinical follow-up. Chin J Intervent Cardiol. 2018;26(10):547-551.

14. Gloekler S, Saw J, Koskinas KC, et al. Left atrial appendage closure for prevention of death, stroke, and bleeding in patients with nonvalvular atrial fibrillation. Int $J$ Cardiol. 2017;249:234-246. doi:10.1016/j.ijcard.2017.08.049

15. Schnabel RB, Yin X, Gona P, et al. 50 year trends in atrial fibrillation prevalence, incidence, risk factors, and mortality in the Framingham Heart Study: a cohort study. Lancet. 2015;386(9989):154-162. doi:10.1016/S0140-6736(14)61774-8

16. Chen ZJ, Yi GW. 2016 ESC guidelines for the management of atrial fibrillation developed. J Clin Cardiol. 2016;32(11):1076-1078.

17. Fauchier L, Cinaud A, Brigadeau F, et al. Device-related thrombosis after percutaneous left atrial appendage occlusion for atrial fibrillation. $J$ Am Coll Cardiol. 2018;71(14):1528-1536. doi:10.1016/j.jacc.2018.01.076

18. Bashir MU, Bhagra A, Kapa S, McLeod CJ. Modulation of the autonomic nervous system through mind and body practices as a treatment for atrial fibrillation. Rev Cardiovasc Med. 2019;20 (3):129-137. doi:10.31083/j.rcm.2019.03.517

19. Figini F, Mazzone P, Regazzoli D, et al. Left atrial appendage closure: a single center experience and comparison of two contemporary devices. Catheter Cardiovasc Interv. 2017;89(4):763-772. doi: $10.1002 / \operatorname{ccd} .26678$ 
20. Bonin M, Mewton N, Roubille F, et al. Effect and safety of morphine use in acute anterior ST-segment elevation myocardial infarction. J Am Heart Assoc. 2018;7(4):e06833. doi:10.1161/ JAHA.117.006833

21. Shen ZF. Clinical curative of new oral anticoagulants for the treatment of gerontal patients with non-valvular atrial fibrillation. Clin Pract Med. 2018;13(18):96-97.

22. Guo YS, Zhang HJ, Li XL, et al. Safety and efficacy of dabigatran in long-term anticoagulation in patients with atrial fibrillation. J Pract Med. 2018;34(19):3277-3279.

23. Kirchhof P, Benussi S, Kotecha D, et al. 2016 ESC Guidelines for the management of atrial fibrillation developed in collaboration with EACTS. Europace. 2016;18(11):1609-1678. doi:10.1093/europace/ euw295
24. Opacic D, van Bragt KA, Nasrallah HM, Schotten U, Verheule S. Atrial metabolism and tissue perfusion as determinants of electrical and structural remodelling in atrial fibrillation. Cardiovasc Res. 2016;109(4):527-541. doi:10.1093/cvr/cvw007

25. Bösche LI, Afshari F, Schöne D, Ewers A, Mügge A, Gotzmann M. Initial experience with novel oral anticoagulants during the first 45 days after left atrial appendage closure with the Watchman device. Clin Cardiol. 2015;38(12):720-724. doi:10.1002/clc.22478

26. Enomoto Y, Gadiyaram VK, Gianni C, et al. Use of non-warfarin oral anticoagulants instead of warfarin during left atrial appendage closure with the Watchman device. Heart Rhythm. 2017;14(1):19-24 doi:10.1016/j.hrthm.2016.10.020

\section{Publish your work in this journal}

Drug Design, Development and Therapy is an international, peerreviewed open-access journal that spans the spectrum of drug design and development through to clinical applications. Clinical outcomes, patient safety, and programs for the development and effective, safe, and sustained use of medicines are a feature of the journal, which has also been accepted for indexing on PubMed Central. The manuscript management system is completely online and includes a very quick and fair peer-review system, which is all easy to use. Visit http://www. dovepress.com/testimonials.php to read real quotes from published authors. 